\title{
Measuring system and method eliminating drifts and parasitic influences for cantilever based bio-sensors
}

\author{
Minchev Gencho $^{1}$, Mincheva Tatiana ${ }^{1}$ and Stavrov Vlado ${ }^{2}$ \\ ${ }^{1}$ Applied Research Unit, Institute of Solid State Physics, Bulgarian Academy of Sciences, 72 Tzarigradsko \\ Chaussee blvd., 1784 Sofia, Bulgaria, Electronic address: MBE@issp.bas.bg; \\ ${ }^{2}$ AMG Technology Ltd., Microelectronics Industrial Zone, PB 43, 2140 Botevgrad, Bulgaria
}

The most natural and universal approach for identification of nano-sized biological objects (macromolecules, molecular complexes, viruses, bacterial spores, etc.) is to immobilize them with respective antibodies on cantilever surface and then to measure the sensor mass change. Difficulties arise due to the ultra small magnitude of these changes. An added to the cantilever mass could be registered as a change in deflection or a shift in resonance frequency. Using only one cantilever as an ultrasensitive balance faces serious obstacles. The frequency change (used as measure for mass change) forms as a sum of many influences (from mass change, but also from temperature, pressure, illumination, etc.) and accumulates several types of drifts (ageing/wearing, degradation of functionalization, irreversible chemical transformations, etc.). Moreover, for the mass-change range of interest those parasitic influences dominate by magnitude.

To overcome the problems we composed a self-consistent solution covering several levels: MEMS construction level, measuring procedures (method) level, data processing level and system level. MEMS structures with four specifically diverged cantilevers for one sensing task, a set of simultaneously taken differential measurements, computing several aggregate quantities from huge amount of precise measurement data and inner for the system continuous calibrations are the key advances.

MEMS cantilever array (figure 1) have been micro-fabricated on double-side polished n-type, $<100\rangle$, Si wafers having Total Thickness Variation (TTV) less than $1 \mu \mathrm{m}$. A standard CMOS compatible process with combined wet $\mathrm{KOH}$ (for back side)/dry (for front side) etching techniques were used. The cantilevers dimensions: sequential length in $309.5 \mu \mathrm{m} \div 320 \mu \mathrm{m}$ range, width $76 \mu \mathrm{m}$, thickness $4 \mu \mathrm{m}$ and $50 \mu \mathrm{m}$ spacing are defined by photolithography. A meander bimorph actuator having thickness of $0.8 \mu \mathrm{m}$ placed over $400 \mathrm{~nm}$ low-stress insulating $\mathrm{SiO}_{2}$ layer was formed by metal deposition/patterning standard process sequence.

The sensor technical realization is presented on figure 2. The frequency of sensor mechanical vibration (if existing) in the resonator is always equal to the excitation frequency. Vibration amplitude conforms to the stored in the resonance energy and is quite big for high $\mathrm{Q}$-factor resonances. At zero phase difference the feeding energy is directly obtainable from the known excitation voltage and the measured amplitude ratio determines the ratio of resonance admittance to the excitation equivalent resistance. At frequencies slightly deviated from resonance frequency the energy accumulated in the resonance is much smaller, loses are also smaller and for restoring the source-sink energy balance a phase shift to the excitation appears that effectively diminishes the energy feed.

The sensor electronics operates in three basic modes, each for a corresponding task. Figure 3 presents the system organization for scanning the range of interest by frequency and locating all existing in that range resonances.

It is the second basic operational mode that permits selecting and locking on each resonance. The system organization for that mode is presented on figure 4.

The first step is for choice of resonance. The system-microcontroller scans the frequency range where the resonance of interest has been located during the previous observable mode. It carries out all this using the first DDS (depicted at the bottom of the figure 4 diagram) through its 32 bit frequency tuning word. The output signal of that first DDS is fed through a x20 PLL to the upper (on figure 4) DDS-structure as clock signal. From this clock signal the DDS-structure produces a frequency twenty-fold lower (equal to the first DDS output frequency - i.e., a frequency equal to the system-microcontroller dictated frequency) and excites the resonator with it. A zero phase offset is added in the intermediate block (between phase accumulator and ROM lock-in table). Thus, the range is scanned transparently through the not interfering second DDS-structure. Selecting the resonance occurs when the phase difference 
becomes zero (or close to zero) and amplitude ratio is at its minimal value (as seen from the upper gain \& phase detector).

Then, as a second step, the system-microcontroller starts to adjust an additional phase offset injected into the DDS-structure (thus changing the excitation phase) together with adjusting the amplitude of the first DDS output signal, until the outputs of the lower gain \& phase detector become zeros - i.e., a condition when no phase difference exists between the resonator vibrations and first DDS output sinusoid together with equality of their amplitudes (zero in logarithmic output scale of gain \& phase detector). This adjustment has to be performed, yet preserving the zero phase difference of excitation. The latter could need a second adjusting loop and continuous frequency tuning for first DDS.

The third step is easy - the system-microcontroller simply switches the x20 PLL input signal from DDS output to the completely equal signal from the resonator. This creates a frequency neutral loop - the signal from the resonator electrodes is fed to the $x 20 \mathrm{PLL}$ where it is multiplied 20 times, then divided 20 times in the phase accumulator and fed back to the resonator. Any shift in center frequency of the resonance is forwarded through this loop and nothing changes. Thus, the system has "locked" on the selected resonance and begins to be continuously driven by that resonance.

Once the sensor electronics is locked-on, a detailed and precise measuring becomes possible. Figure 5 presents the configuration for making such measurements - i.e., the third basic operational mode. In fact, it is the configuration that appears after performing the three steps from the previous mode.

A 256 channel system (figure 6) has dimensions of $20 \times 85 \times 175 \mathrm{~mm}$. The cantilever array structures are mounted on washer-like holders. These holders are staked sequentially and form a hermetic tube through which the carrier fluid is pumped.

A research instrument is shown on figure 7 . It is an USB powered complete "virtual" instrument with versatile user interface and direct programming from user PC. It excites the cantilever structure with $5 \mathrm{ppb}$ frequency resolution and $0.1 \mathrm{ppm}$ frequency absolute accuracy and measures the cantilever responses within $105 \mathrm{~dB}$ dynamic range to their thermal noise limits with $0.06 \mathrm{~dB}$ resolution. The "standalone" sensors (fig. 2) achieve $0.1 \mathrm{pg}$ interchannel sensitivity and $10 \mathrm{pg}$ absolute calibrations. The multichannel system (fig. 3 ) achieves $10 \mathrm{fg}\left(10^{-14} \mathrm{~g}\right)$ interchannel and $0.25 \mathrm{pg}$ absolute resolution.

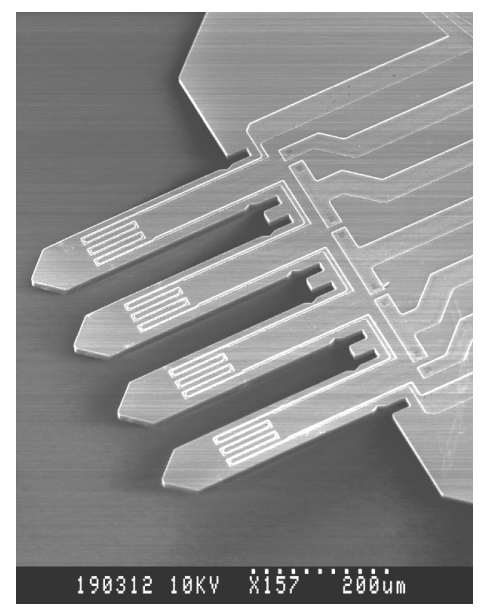

FIG. 1: Piezoresistive sensor array.

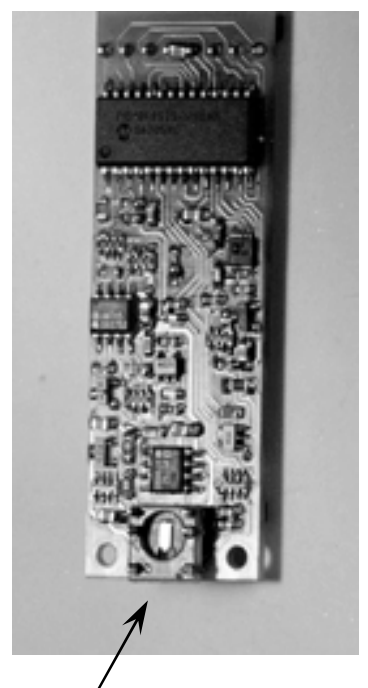

FIG. 2: "Stand-alone" intelligent sensor (1 to 16 channels) with CAN/LIN interfaces. 


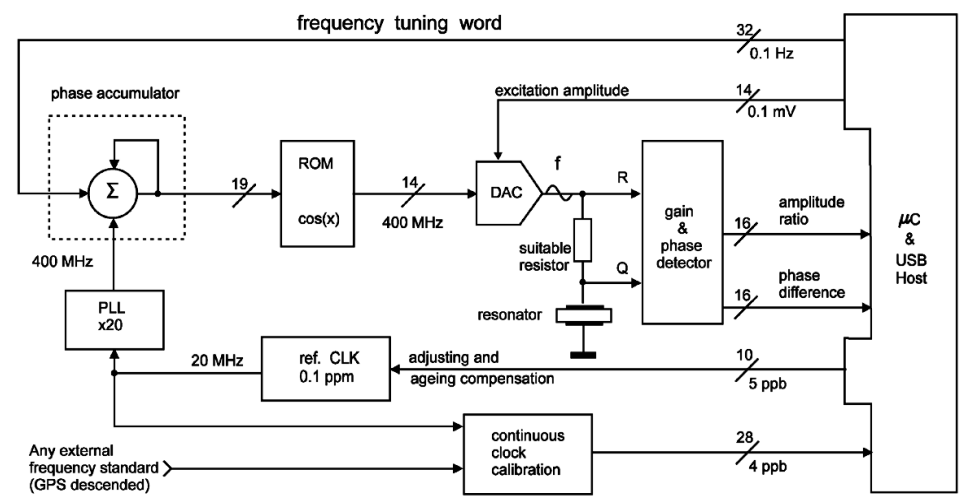

FIG. 3: Setup for measuring the resonator response to the excitation frequency. The setup could be functionally divided into three subsystems: i) precise gain \& phase measuring; ii) phase lock loop, reference clock and clock calibration; iii) phase accumulator, read-only-memory containing lookin-table of $\cos (\mathrm{x})$ function and output DAC generating the excitation sinusoid (a kind of direct digital synthesizer). Scanning is performed by the 32 bit ,frequency tuning word“ with of $0.1 \mathrm{~Hz}$ minimal step.

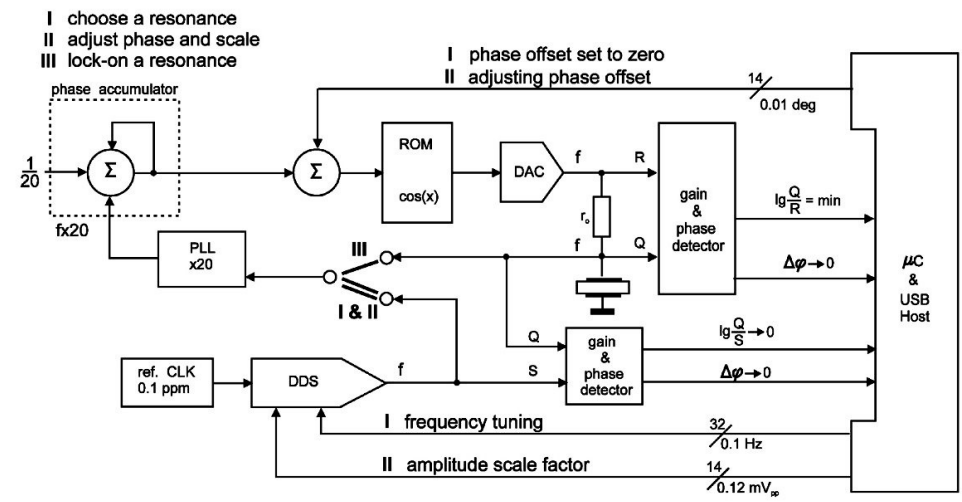

FIG. 4: The configuration for the three steps procedure of resonance selection. In this configuration there are two gain \& phase detectors and two direct digital synthesizers (DDS). The three steps are marked I, II and III.

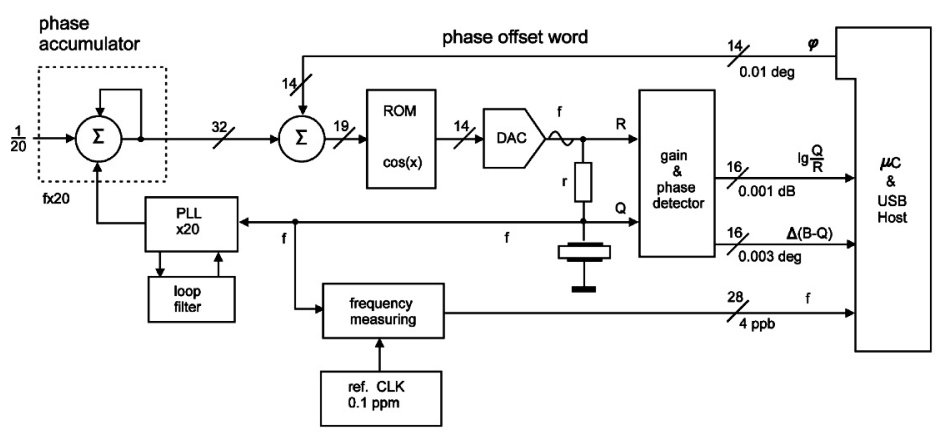

FIG. 5: Configuration for measuring a resonance. The dictated from system-microcontroller 14 bit phase offset word is summed with the fourteen most significant bits of the 32 bit word from phase accumulator and produces, after truncation, a 19 bit index for the look-in-table in ROM. The table contains 14 bit values of $\cos (x)$ function that are sent to the output DAC. Amplitude ratio and phase difference are measured by 16 bit $A D C$-system and the current frequency is measured by a hardware block with 28 bit resolution and $0.1 \mathrm{ppm}$ absolute accuracy. 


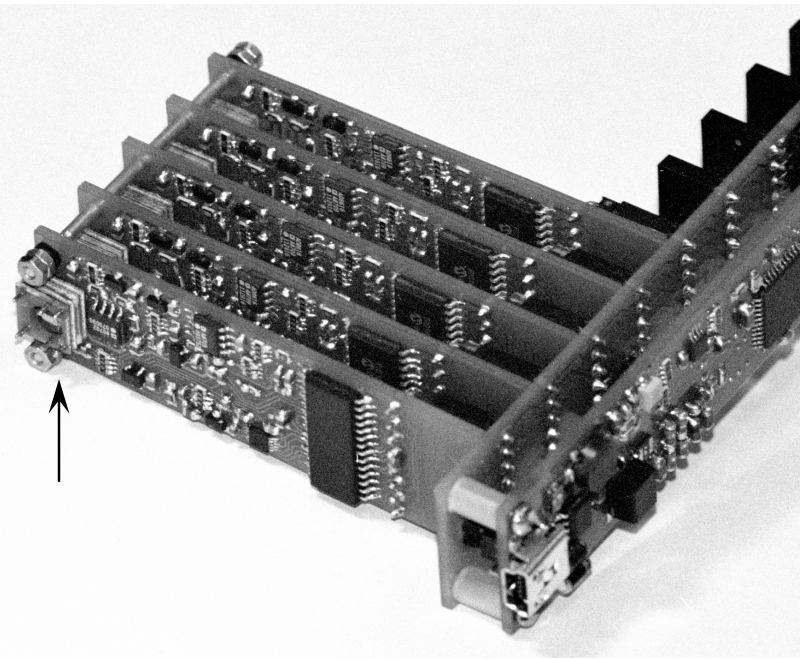

FIG. 6: USB powered multi-channel "spectroscopic type" system (channels number not limited).

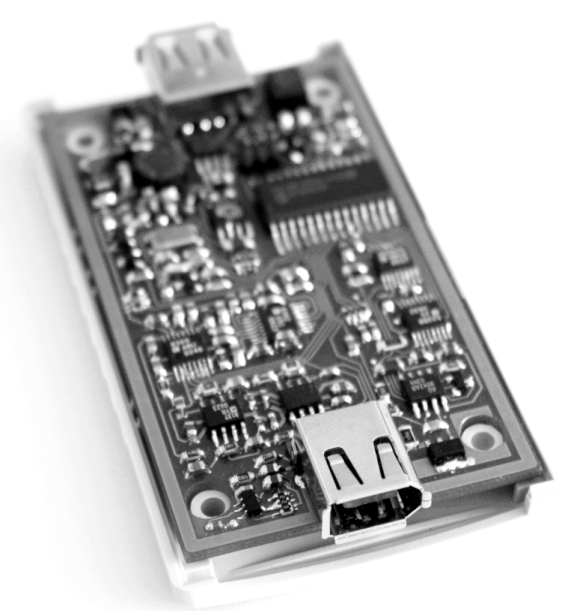

FIG. 7: Precise development system for cantilever sensor functionalizing. 